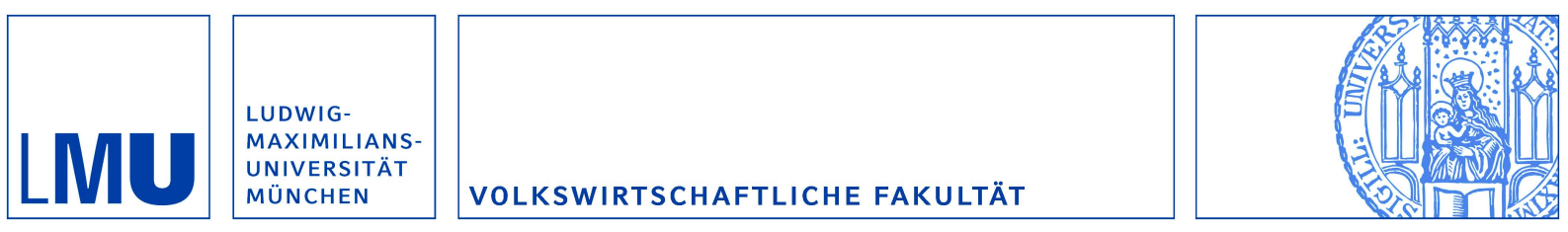

Schlicht, Ekkehart:

Estimating the Smoothing Parameter in the So-Called Hodrick-Prescott Filter

Munich Discussion Paper No. 2004-2

Department of Economics

University of Munich

Volkswirtschaftliche Fakultät

Ludwig-Maximilians-Universität München

Online at https://doi.org/10.5282/ubm/epub.304 


\title{
ESTIMATING THE SMOOTHING PARAMETER IN THE SO-CALLED HODRICK-PRESCOTT FILTER
}

\author{
EKKEHART SCHLICHT
}

\begin{abstract}
This note gives a fairly complete statistical description of the Hodrick-Prescott Filter (1997), originally proposed by Leser (1961). It builds on an approach to seasonal adjustment suggested by Leser (1963) and Schlicht (1981, 1984). A moments estimator for the smoothing parameter is proposed that is asymptotically equivalent to the maximumlikelihood estimator, has a straightforward intuitive interpretation and is more appropriate for short series than the maximum-likelihood estimator. The method is illustrated by an application and several simulations.
\end{abstract}

Acknowledgement. I thank Johannes Ludsteck for several helpful suggestions and for providing the Mathematica Package (Ludsteck, 2004) used for the computations in this paper.

Key words and phrases. Hodrick-Prescott filter, Kalman filtering, Kalman-Bucy, WhittakerHenderson graduation, spline, state-space models, random walk, time-varying coefficients, adaptive estimation, time-series, seasonal adjustment, trend.

Journal of Economic Literature classification numbers: $\mathrm{C}_{22}$. 


\section{INTRODUCTION}

What is known as the Hodrick-Prescott Filter (1997) is widely used in applications and has been embodied in various statistical packages. King and Rebelo $(1993,230)$ write that the filter "is commonly used in investigations of the stochastic properties of real business cycle models," and many papers have been published that either use or improve the filter. The economics databank EconLit lists 72 papers with "Hodrick-Prescott Filter" in the title or abstract, and the statistic programs eViews and Stata provide the filter as a standard feature.

The filter has been proposed originally by Leser (1961), building on the graduation method developed by Whittaker (1923) and Henderson (1924). It requires a smoothing constant as an input. This constant is usually fixed in an ad-hoc way. The program eViews recommends 100 for annual data, 1600 for quarterly data, and 14.400 for monthly data, for instance, presumably summung up various findings in simulation studies and applied research. A theoretical approach to the determination of the filter has been suggested by Hodrick and Prescott $(1997,4)$ who referred to Kalmanfiltering and related the smoothing constant to a ratio of variances. (Their guess of a variance ratio of 1600 for quarterly data established a custom.) Earlier, Schlicht $(1981,1984)$ proposed a two-sided filter, also based on the variance ratio as a smoothing constant. Although it is possible, in principle, to estimate the variance ratios both in the Kalman and the Schlicht framework by using maximum-likelihood estimates, practical implementation is often not satisfactory, as corner solutions tend to occur for shorter time series, and the rule-of thumb approach prevails.

The aim of this paper is to offer a rather systematic exposition and a straightforward method for estimating the smoothing constant, based on the approach by Schlicht (1984). A maximum likelihood estimator and a related and more intuitive a moments estimator will be derived and compared. A simulation study illustrates the performance of these estimators.

The paper is organized as follows. In Section 1 the filter is described; in Section 2, a statistical interpretation of the filter is given that involves some formal parameters. Section 3 gives the estimator for those parameters. In Section 4 it is proven that the descriptive procedure described in Section 
1 gives an unbiased maximum-likelihood estimate for the trend, given a smoothing parameter.

Given any smoothing parameter, the covariance matrix of the trend estimate is given in Section 5 . Section 6 turns to estimation of the variances by a maximum likelihood method. The variances determine the smoothing parameter. It is shown that the numerical problem can be simplified considerably in several ways.

Section 7 describes a moments estimator for the variances. This estimator is characterized by the property that the computed variances of the error terms are equal to their expectations. In Section 8 it is shown that the likelihood estimates and the moments estimates differ only slightly and approach each other with an increasing length of the time series. This gives intuitive appeal to the maximum likelihood estimator and statistical appeal to the moments estimator.

Section 9 comments on some practical aspects and presents some simulations and section 10 points out some open problems.

\section{THE FiLteR}

Consider a time series $x \in \Re^{T}$ that is to be decomposed into a trend $y \in \Re^{T}$ and an irregular component $u \in \Re^{T}$ :

$$
x=y+u
$$

Define the trend disturbance $v \in \Re^{T-1}$ as

$$
v_{t}=\left(\left(y_{t}-y_{t-1}\right)-\left(y_{t-1}-y_{t-2}\right)\right) \quad t=3,4, \ldots, T
$$

or

$$
v=P y
$$

with

$$
P:=\left(\begin{array}{cccccc}
1 & -2 & 1 & & & 0 \\
& 1 & -2 & 1 & & \\
& & \cdot & \cdot & \cdot & \\
0 & & & 1 & -2 & 1
\end{array}\right)
$$

of order $(T-2) \times T$. 
The decomposition of the original series $x$ into trend $y$ and irregular component $u$ is obtained by minimzing the weighted sum of squares

$$
u^{\prime} u+\alpha \cdot v^{\prime} v=(x-y)^{\prime}(x-y)+\alpha \cdot y^{\prime} P^{\prime} P y
$$

with respect to $y$. This gives the first-order condition

$$
\left(I+\alpha \cdot P^{\prime} P\right) y=x
$$

As $\left(I+\alpha P^{\prime} P\right)$ is positive definite, the second order condition is satisfied in any case.

The the system matrix in (5) can be written as as

$$
\left(\begin{array}{ll}
I, & \alpha^{\frac{1}{2}} P^{\prime}
\end{array}\right)\left(\begin{array}{c}
I \\
\alpha^{\frac{1}{2}} P
\end{array}\right)
$$

As $\left(I, \quad \alpha^{\frac{1}{2}} P^{\prime}\right)$ is of full rank, $\left(I+\alpha \cdot P^{\prime} P\right)$ is nonsingular and equation (5) has the unique solution

$$
y=\left(I+\alpha P^{\prime} P\right)^{-1} x
$$

Equation (6) defines the descriptive filter that associates a trend $y$ to the time series $x$, depending on the smoothing parameter $\alpha$.

\section{STOCHASTIC INTERPRETATION}

Equations (1) and (2) can be embedded in a stochastic model by assuming that the disturbances $u$ and $v$ in (1) and (2) are normal random variables with variances $\sigma_{u}^{2}$ and $\sigma_{v}^{2}$ and zero expectations:

$$
u \sim \mathcal{N}\left(0, \sigma_{u}^{2}\right), \quad v \sim \mathcal{N}\left(0, \sigma_{v}^{2}\right)
$$

This turns $x$ and $y$ into random variables with probability distributions that will be derived in the following.

As the rank of $P$ is $T-2$, there exist two orthogonal solutions $z$ to the equation $P z=0$. These can be taken as columns of a $(T \times 2)$-matrix $Z$ that satisfies

$$
P Z=0, Z^{\prime} Z=0
$$

Consider now

$$
\left(\begin{array}{l}
P \\
Z^{\prime}
\end{array}\right)\left(\begin{array}{ll}
P^{\prime} & Z
\end{array}\right)=\left(\begin{array}{cc}
P P^{\prime} & 0 \\
0 & I
\end{array}\right)
$$


which is of full rank. Inverting both sides of (9), pre-multiplying by $\left(P^{\prime}, Z\right)$ and multiplying from the right-hand side by $\left(\begin{array}{c}P \\ Z^{\prime}\end{array}\right)$ implies

$$
\left(\begin{array}{ll}
P^{\prime} & Z
\end{array}\right)\left(\begin{array}{cc}
\left(P P^{\prime}\right)^{-1} & 0 \\
0 & I
\end{array}\right)\left(\begin{array}{l}
P \\
Z^{\prime}
\end{array}\right)=I
$$

and hence

$$
P^{\prime}\left(P P^{\prime}\right)^{-1} P+Z Z^{\prime}=I
$$

The auxiliary matrix $Z$ comprises the set of the two orthogonal solutions to the equation $P z=0$. Any solution to (2) can be witten as

$$
y=P^{\prime}\left(P P^{\prime}\right)^{-1} v+Z \beta
$$

with $\beta \in \Re^{2}$ as a formal parameter vector. The time series $x$ can be seen as the sum of $y$ and $u$, and hence as brought about by the disturbances $u$ and $v$ :

$$
x=u+P^{\prime}\left(P P^{\prime}\right)^{-1} v+Z \beta
$$

Combining (12) and (13) gives

$$
\left(\begin{array}{l}
x \\
y
\end{array}\right)=\left(\begin{array}{ll}
I & P^{\prime}\left(P P^{\prime}\right)^{-1} \\
0 & P^{\prime}\left(P P^{\prime}\right)^{-1}
\end{array}\right)\left(\begin{array}{l}
u \\
v
\end{array}\right)+\left(\begin{array}{c}
Z \\
Z
\end{array}\right) \beta
$$

As the disturbances $u$ and $v$ are normal with variances $\sigma_{u}^{2}$ and $\sigma_{v}^{2}$, the vector $\left(u^{\prime}, v^{\prime}\right)$ is normal as well:

$$
\left(\begin{array}{c}
u \\
v
\end{array}\right) \sim \mathcal{N}\left(0, S_{u v}\right)
$$

The co-variance matrix is

$$
S_{u v}:=\left(\begin{array}{cc}
\sigma_{u}^{2} \cdot I & 0 \\
0 & \sigma_{v}^{2} \cdot I
\end{array}\right)
$$

From (14) to (16) we obtain

$$
\left(\begin{array}{l}
x \\
y
\end{array}\right) \sim \mathcal{N}\left(\left(\begin{array}{c}
Z \\
Z
\end{array}\right) \beta, S_{x y}\right)
$$


with $^{1}$

$$
S_{x y}:=\left(\begin{array}{cc}
\sigma_{u}^{2} I+\sigma_{v}^{2} Q & \sigma_{v}^{2} Q \\
\sigma_{v}^{2} Q & \sigma_{v}^{2} Q
\end{array}\right)
$$

and

$$
Q:=P^{\prime}\left(P P^{\prime}\right)^{-1}\left(P P^{\prime}\right)^{-1} P
$$

Note that (8) entails

$$
Z^{\prime} Q=0
$$

From (17) we obtain the marginal density of $x$ as

$$
x \sim \mathcal{N}\left(Z \beta, S_{x}\right)
$$

with

$$
\begin{aligned}
S_{x} & :=\left(\sigma_{u}^{2} I+\sigma_{v}^{2} Q\right) \\
& =\sigma_{v}^{2}\left(I+\frac{\sigma_{v}^{2}}{\sigma_{u}^{2}} Q\right)
\end{aligned}
$$

Note that

$$
\begin{aligned}
Z^{\prime} S_{x}^{-1} & =\frac{1}{\sigma_{v}^{2}} Z^{\prime}\left(I-\frac{\sigma_{v}^{2}}{\sigma_{u}^{2}} Q+\left(\frac{\sigma_{v}^{2}}{\sigma_{u}^{2}} Q\right)^{2}-\left(\frac{\sigma_{v}^{2}}{\sigma_{u}^{2}} Q\right)^{3}+\left(\frac{\sigma_{v}^{2}}{\sigma_{u}^{2}} Q\right)^{4}-\ldots\right) \\
& =\frac{1}{\sigma_{v}^{2}} Z^{\prime}
\end{aligned}
$$

The marginal density of $y$ for given $x$ is

$$
(y \mid x) \sim \mathcal{N}\left(\bar{y}, S_{y}\right)
$$

where

$$
\begin{aligned}
\bar{y} & :=Z \beta+\sigma_{v}^{2} Q\left(\sigma_{u}^{2} I+\sigma_{v}^{2} Q\right)^{-1}(x-Z \beta) \\
S_{y \mid x} & :=\sigma_{v}^{2} Q-\sigma_{v}^{2} Q\left(\sigma_{u}^{2} I+\sigma_{v}^{2} Q\right)^{-1} \sigma_{v}^{2} Q
\end{aligned}
$$

Equations (25) and (26) can be simplified. Note that

$$
I-\sigma_{v}^{2}\left(\sigma_{u}^{2} I+\sigma_{v}^{2} Q\right)^{-1} Q=\sigma_{u}^{2}\left(\sigma_{u}^{2} I+\sigma_{v}^{2} Q\right)^{-1}
$$

\footnotetext{
${ }^{1}$ Note that the covariance matrix $S_{x y}$ is not of full rank. Hence $\left(x^{\prime}, y^{\prime}\right)$ is distributed on a subspace of $\Re^{2 T}$ that is determined by the parameter $\lambda$.
} 
which is verified by pre-multiplication with $\left(\sigma_{u}^{2} I+\sigma_{v}^{2} Q\right)$. Hence the covariance matrix can be written as

$$
S_{y \mid x}=\sigma_{u}^{2} \sigma_{v}^{2} Q\left(\sigma_{u}^{2} I+\sigma_{v}^{2} Q\right)^{-1}
$$

With $\alpha=\sigma_{u}^{2} / \sigma_{v}^{2}$ we have

$$
\sigma_{v}^{2} Q\left(\sigma_{u}^{2} I+\sigma_{v}^{2} Q\right)^{-1}=Q(\alpha I+Q)^{-1}
$$

and with (11)

$$
\begin{aligned}
(\alpha I+Q)\left(P^{\prime} P\right) & =\left(\alpha P^{\prime} P+P^{\prime}\left(P P^{\prime}\right)^{-1} P\right) \\
& =\left(I+\alpha P^{\prime} P-Z Z^{\prime}\right)
\end{aligned}
$$

Hence

$$
(\alpha I+Q)^{-1}=\left(P^{\prime} P\right)\left(I+\alpha P^{\prime} P-Z Z^{\prime}\right)^{-1}
$$

and

$$
\sigma_{v}^{2} Q\left(\sigma_{u}^{2} I+\sigma_{v}^{2} Q\right)^{-1}=\left(I-Z Z^{\prime}\right)\left(I+\alpha P^{\prime} P-Z Z^{\prime}\right)^{-1}
$$

With (27), and (28), (25) can be re-written as

$$
\bar{y}=Z \beta+Q(\alpha I+Q)^{-1}(x-Z \beta)
$$

or

$$
\bar{y}=Z \beta+\left(I-Z Z^{\prime}\right)\left(I+\frac{\sigma_{u}^{2}}{\sigma_{v}^{2}} P^{\prime} P-Z Z^{\prime}\right)^{-1}(x-Z \beta)
$$

Further we have

$$
\begin{aligned}
P^{\prime} P Q & =\frac{\sigma_{u}^{2}}{\sigma_{v}^{2}} P^{\prime} P+P^{\prime}\left(P P^{\prime}\right)^{-1} P \\
& =I+\frac{\sigma_{u}^{2}}{\sigma_{v}^{2}} P^{\prime} P-Z Z^{\prime}
\end{aligned}
$$

and hence

$$
S_{y \mid x}=\sigma_{u}^{2} Q\left(I+\frac{\sigma_{u}^{2}}{\sigma_{v}^{2}} Q\right)^{-1}
$$

or

$$
S_{y \mid x}=\left(I-\left(I-Z Z^{\prime}\right)\left(I+\frac{\sigma_{u}^{2}}{\sigma_{v}^{2}} P^{\prime} P-Z Z^{\prime}\right)^{-1}\right) \sigma_{v}^{2} Q
$$




\section{Estimating the Formal Parameters $\beta$}

The parameters that need to be estimated are the formal parameter vector $\beta$ and the variances $\sigma_{u}^{2}$ and $\sigma_{v}^{2}$.

The estimation of the formal parameters $\beta$ is straigtforward. Equation (21) gives rise to the likelihood function

$$
\begin{aligned}
L\left(x, \beta, \sigma_{u}^{2}, \sigma_{v}^{2}\right):= & -\log \operatorname{det}\left(\sigma_{u}^{2} I+\sigma_{v}^{2} Q\right) \\
& -(x-Z \beta)^{\prime}\left(\sigma_{u}^{2} I+\sigma_{v}^{2} Q\right)^{-1}(x-Z \beta)
\end{aligned}
$$

Minimizing $L$ with respect to $\beta$ leads to sufficient condition

$$
Z^{\prime}\left(\sigma_{u}^{2} I+\sigma_{v}^{2} Q\right)^{-1} x=Z^{\prime}\left(\sigma_{u}^{2} I+\sigma_{v}^{2} Q\right)^{-1} Z \hat{\beta}
$$

In view of $(22),(23)$, and (8) this reduces to

$$
\hat{\beta}=Z^{\prime} x
$$

\section{Estimating The TREND $y$}

If we substitute the formal parameters $\beta$ with the estimator $\hat{\beta}$ in (35), we obtain the conditional distribution of the trend $y$ (which is a random variable). It seems sensible to take the expectation of this random variable as our estimator for the trend. This yields:

$$
\hat{y}:=Z \hat{\beta}+Q\left(\frac{\sigma_{u}^{2}}{\sigma_{v}^{2}} I+Q\right)^{-1}(x-Z \hat{\beta})
$$

Theorem 1. With the smoothing constant $\alpha$ equal to the variance ratio $\sigma_{u}^{2} / \sigma_{v}^{2}$ the descriptive decomposition (6) is numerically identical to the estimator (36).

Proof. Setting $\alpha=\sigma_{u}^{2} / \sigma_{v}^{2}$ in (36) and and ordering terms gives

$$
\hat{y}=Z \hat{\beta}+Q(\alpha I+Q)^{-1}(x-Z \hat{\beta})
$$

Note that

$$
Q(\alpha I+Q)^{-1}=I-\alpha(\alpha I+Q)^{-1}
$$

which is verified by right-hand multiplication with $(\alpha I+Q)$. Inserting this into (37) and re-arranging terms gives

$$
(\alpha I+Q)^{-1}(x-Z \hat{\beta})=\frac{1}{\alpha}(x-\hat{y})
$$


This can be inserted into (37) again, and we obtain

$$
\hat{y}=Z \hat{\beta}+Q \frac{1}{\alpha}(x-\hat{y})
$$

Pre-multiplication with $\alpha P^{\prime} P$ yields

$$
\alpha P^{\prime} P \hat{y}=\alpha P^{\prime} P Z \hat{\beta}+P^{\prime} P Q(x-\hat{y})
$$

As $P Z=0$, the first term on the right-hand side cancels. From the definition (19) of $Q$ and (11) it follows that

$$
P^{\prime} P Q=I-Z Z^{\prime}
$$

Substituting this into (41) gives

$$
\alpha P^{\prime} P \hat{y}=(x-\hat{y})-Z Z^{\prime}(x-\hat{y})
$$

Because of (35) we have $Z^{\prime} x=\hat{\beta}$. Pre-multiplying (40) by $Z^{\prime}$ while noting that $Z^{\prime} Z=I$ and $Z^{\prime} Q=0$ results in $Z^{\prime} \hat{y}=\hat{\beta}$ as well. Hence the last term in (43) cancels and we obtain

$$
\left(I+\alpha P^{\prime} P\right) \hat{y}=x
$$

which is numerically identical to the normal equation (5) that defines the descriptive filter.

\section{THE Covariance Matrix OF the Estimates}

Consider a given time series $x$ and a realization of the associated trend $y$. Because $x$ can be viewed as brought about as the sum of the trend $y$ and the disturbance $u$, we can write :

$$
\hat{y}=\left(I+\alpha P^{\prime} P\right)^{-1}(y+u)
$$

Since

$$
y=\left(I+\alpha P^{\prime} P\right) y-\alpha P^{\prime} P y
$$

and $v=P y$, equation (45) can be written as

$$
\hat{y}-y=\left(I+\alpha P^{\prime} P\right)^{-1}\left(u-\alpha P^{\prime} v\right)
$$

Equation (47) gives the estimation error, and the covariance matrix of this error is calculated as

$$
E\left\{(\hat{y}-y)^{\prime}(\hat{y}-y)\right\}=\sigma_{u}^{2}\left(I+\alpha P^{\prime} P\right)^{-1}
$$


For given variances (and therefore a given smoothing constant $\alpha=\sigma_{u}^{2} / \sigma_{v}^{2}$ ), equation (48) gives the variances of the trend estimates. The square roots of the main diagonal elements of (48) give the standard errors of the corresponding point estimates $\hat{y}_{t}$ of the trend. It is thus possible to guess, for any smoothing parameter $\alpha$, the precision of the trend estimate.

\section{Maximum-Likelihood Estimation of the VARIANCeS}

In order to estimate the smoothing parameter $\alpha$, we turn now to estimating the variances $\sigma_{u}^{2}$ and $\sigma_{v}^{2}$. A first approach is to simply write down the maximum likelihood function. The distribution of the observations $x$ is given by density function (21). Taking logarithms and disregarding constants gives the likelikood

$$
\begin{aligned}
L\left(x, \lambda, \sigma_{u}^{2}, \sigma_{v}^{2}\right):= & -\log \operatorname{det}\left(\sigma_{u}^{2} I+\sigma_{v}^{2} Q\right) \\
& -(x-Z \beta)^{\prime}\left(\sigma_{u}^{2} I+\sigma_{v}^{2} Q\right)^{-1}(x-Z \beta)
\end{aligned}
$$

By replacing the parameter $\lambda$ with its estimate $\hat{\lambda}=Z^{\prime} x$ from (35), we obtain the concentrated likelihood

$$
\begin{aligned}
L^{*}\left(x, \sigma_{u}^{2}, \sigma_{v}^{2}\right):= & -\log \operatorname{det}\left(\sigma_{u}^{2} I+\sigma_{v}^{2} Q\right) \\
& -x^{\prime}\left(I-Z Z^{\prime}\right)^{\prime}\left(\sigma_{u}^{2} I+\sigma_{v}^{2} Q\right)^{-1}\left(I-Z Z^{\prime}\right) x \quad(50)
\end{aligned}
$$

This would suffice, in principle, to estimate the variances $\sigma_{u}^{2}$ and $\sigma_{v}^{2}$, but the problem can be simplified considerably. The following theorem states that the likelihood (50) can be expressed in terms of the estimated trend $\hat{y}$ and the weighted sum of the variances of the estimates errors $\hat{u}$ and $\hat{v}$ which are defined as follows:

$$
\begin{aligned}
\hat{y} & :=\left(I-\alpha P^{\prime} P\right)^{-1} x \\
\hat{u} & :=x-\hat{y} \\
\hat{v} & :=P \hat{y}
\end{aligned}
$$

Theorem 2. The likelihood (50) can be written as

$$
L^{*}\left(x, \sigma_{u}^{2}, \sigma_{v}^{2}\right)=-\log \operatorname{det}\left(\sigma_{u}^{2} I+\sigma_{v}^{2} Q\right)-\frac{1}{\sigma_{u}^{2}} \hat{u}^{\prime} \hat{u}-\frac{1}{\sigma_{v}^{2}} \hat{v}^{\prime} \hat{v}
$$

Proof. As the first terms of eq (50) and (54) are identical, it suffices to show that the quadratic forms in these equations are the same. Consider first the 
quadratic in (54). From (51) we obtain

$$
\hat{u}^{\prime} \hat{u}=x^{\prime}\left(I-\left(I+\alpha P^{\prime} P\right)^{-1}\right)\left(I-\left(I+\alpha P^{\prime} P\right)^{-1}\right) x
$$

and

$$
\hat{v}^{\prime} \hat{v}=x^{\prime}\left(I+\alpha P^{\prime} P\right)^{-1} P^{\prime} P\left(I+\alpha P^{\prime} P\right)^{-1} x
$$

Because

$$
\left(I+\alpha P^{\prime} P\right)^{-1}=I-\alpha P^{\prime} P+\left(\alpha P^{\prime} P\right)^{2}-\left(\alpha P^{\prime} P\right)^{3}+\ldots
$$

the matrices $P^{\prime} P$ and $\left(I+\alpha P^{\prime} P\right)^{-1}$ commute and we can re-write equation $(56)$ as

$$
\hat{v}^{\prime} \hat{v}=x^{\prime} P^{\prime} P\left(I+\alpha P^{\prime} P\right)^{-1}\left(I+\alpha P^{\prime} P\right)^{-1} x
$$

Combining (54) and $(56)$ gives

$$
\hat{u}^{\prime} \hat{u}+\alpha \hat{v}^{\prime} \hat{v}=x^{\prime}\left(I-\left(I+\alpha P^{\prime} P\right)^{-1}\right) x
$$

With

$$
A:=\left(I-\left(I+\alpha P^{\prime} P\right)^{-1}\right)
$$

and $\alpha=\sigma_{u}^{2} / \sigma_{v}^{2}$ the quadratic in equation (54) is

$$
\frac{1}{\sigma_{u}^{2}} \hat{u}^{\prime} \hat{u}+\frac{1}{\sigma_{v}^{2}} \hat{v}^{\prime} \hat{v}=\frac{1}{\sigma_{u}^{2}} x^{\prime} A x
$$

Consider next the quadratic in (50). With

$$
B:=\left(I-Z Z^{\prime}\right)^{\prime}(\alpha I+Q)^{-1}\left(I-Z Z^{\prime}\right)
$$

it is

$$
x^{\prime}\left(I-Z Z^{\prime}\right)^{\prime}\left(\sigma_{u}^{2} I+\sigma_{v}^{2} Q\right)^{-1}\left(I-Z Z^{\prime}\right) x=\frac{1}{\sigma_{v}^{2}} x^{\prime} B x
$$

Right-hand multiplication of (59) by the non-singular matrices $\left(I+\alpha P^{\prime} P\right)$ and $(\alpha I+Q)$ and use of (11) results in

$$
A\left(I+\alpha P^{\prime} P\right)(\alpha I+Q)=\alpha\left(I+\alpha P^{\prime} P-Z Z^{\prime}\right)
$$

Equation (61) can be re-written as

$$
B=(\alpha I+Q)^{-1}-\frac{1}{\alpha} Z Z^{\prime}
$$


This makes use of the fact that the matrices $\left(I-Z Z^{\prime}\right)$ and $(\alpha I+Q)^{-1}$ commute, that $\left(I-Z Z^{\prime}\right)$ is idempotent and that $Z^{\prime} Q=0$. Righ-hand multiplication of (64) by $(\alpha I+Q)$ and $\left(I+\alpha P^{\prime} P\right)$ yields

$$
B\left(I+\alpha P^{\prime} P\right)(\alpha I+Q)=I+\alpha P^{\prime} P-Z Z^{\prime}
$$

This makes use of the fact that the non-singular matrices $\left(I+\alpha P^{\prime} P\right)$ and $(\alpha I+Q)$ commute and that $P Z=0$. Equations (63) and (65) imply

$$
\frac{1}{\sigma_{u}^{2}} A=\frac{1}{\sigma_{v}^{2}} B
$$

Therefore the expressions given in equations (60) and (62) are identical.

For purposes of estimation, it is useful to parametrize the likelihood function (54) by $\alpha$ and $\sigma_{u}^{2}$ instead of $\sigma_{u}^{2}$ an $\sigma_{v}^{2}$. Because $\sigma_{v}^{2}=\sigma_{u}^{2} / \alpha$, we can write:

$$
\begin{aligned}
L^{* *}\left(x, \sigma_{u}^{2}, \alpha\right):= & -\log \operatorname{det}(\alpha I+Q)-\frac{1}{\sigma_{u}^{2}}\left(\hat{u}^{\prime} \hat{u}+\alpha \hat{v}^{\prime} \hat{v}\right) \\
& +T \cdot \log \alpha-T \cdot \log \sigma_{u}^{2}
\end{aligned}
$$

For any given $\alpha$, the minimization of $L^{* *}$ with repect to $\sigma_{u}^{2}$ leads to the necessary and sufficient conditions

$$
\begin{aligned}
\frac{\partial L^{* *}}{\partial \sigma_{u}^{2}} & =-\frac{T}{\sigma_{u}^{2}}+\frac{1}{\sigma_{u}^{4}}\left(\hat{u}^{\prime} \hat{u}+\alpha \hat{v}^{\prime} \hat{v}\right)=0 \\
\left.\frac{\partial^{2} L^{* *}}{\partial\left(\sigma_{u}^{2}\right)^{2}}\right|_{\frac{L_{L}^{* *}}{\partial \sigma_{u}^{2}}=0} & =-\frac{T}{\sigma_{u}^{4}}<0
\end{aligned}
$$

which imply the estimator

$$
\hat{\sigma}_{u}^{2}=\frac{1}{T}\left(\hat{u}^{\prime} \hat{u}+\alpha \hat{v}^{\prime} \hat{v}\right)
$$

for the variance of $u$.

Given any smoothing parameter $\alpha$, equation (56) permits estimating the precision of the trend estimates - the covariance matrix (48) - in terms of the calculated errors:

$$
E\left\{(\hat{y}-y)^{\prime}(\hat{y}-y)\right\}=\frac{1}{T}\left(\hat{u}^{\prime} \hat{u}+\alpha \hat{v}^{\prime} \hat{v}\right)\left(I+\alpha \cdot P^{\prime} P\right)^{-1}
$$


By inserting (70) into (67) and disregarding constants, a concentrated likelihood function can be derived that involves the smoothing parameter $\alpha$ as its only parameter:

$$
L^{* * *}(x ; \alpha):=-\log \operatorname{det}(\alpha I+Q)-T \cdot \log R(\alpha)+T \cdot \log \alpha \quad(72)
$$

with

$$
R(\alpha):=\hat{u}^{\prime} \hat{u}+\alpha \hat{v}^{\prime} \hat{v}
$$

With (72), maximum likelihood estimation reduces to maximizing over just one parameter. As the solution $\hat{y}$ to the band-diagonal normal equation (6) is straightforward, minimization of $L^{* * *}$ with respect to the smoothing parameter $\alpha$ can be performed numerically. The solution $\hat{y}$ can be calculated for any $\alpha$. The value of $R(\alpha)$ is calculated via (51) - (53) and (73). For any $\alpha$, the corresponding variances are computed according to (70) and $\alpha=\sigma_{u}^{2} / \sigma_{v}^{2}$ as

$$
\begin{aligned}
\hat{\sigma}_{u}^{2} & =\frac{1}{T} R(\alpha) \\
\hat{\sigma}_{v}^{2} & =\frac{1}{T} \frac{R(\alpha)}{\alpha}
\end{aligned}
$$

The likelihood function can be further simplified with respect to the first term. Consider

$$
(\alpha I+Q)\left(\begin{array}{cc}
P^{\prime} & Z
\end{array}\right)\left(\begin{array}{c}
P \\
Z^{\prime}
\end{array}\right)=\left(\alpha P^{\prime} P+\alpha Z Z^{\prime}+I-Z Z^{\prime}\right)
$$

As

$$
\begin{aligned}
\operatorname{det}\left(\left(\begin{array}{ll}
P^{\prime} & Z
\end{array}\right)\left(\begin{array}{l}
P \\
Z^{\prime}
\end{array}\right)\right) & =\operatorname{det}\left(\left(\begin{array}{c}
P \\
Z^{\prime}
\end{array}\right)\left(\begin{array}{ll}
P^{\prime} & Z
\end{array}\right)\right) \\
& =\operatorname{det}\left(\begin{array}{cc}
P P^{\prime} & 0 \\
0 & I
\end{array}\right) \\
& =\operatorname{det}\left(P P^{\prime}\right)
\end{aligned}
$$

and $(76)$ implies

$$
\operatorname{det}\left(P P^{\prime}\right) \operatorname{det}(\alpha I+Q)=\operatorname{det}\left(I+\alpha P^{\prime} P+(\alpha-1) Z Z^{\prime}\right)
$$


Right-hand multiplication by $\operatorname{det}\left(I+\alpha P^{\prime} P\right)^{-1}$ gives

$$
\begin{aligned}
\operatorname{det}\left(P P^{\prime}\right)(\alpha I+Q) & \operatorname{det}\left(I+\alpha P^{\prime} P\right)^{-1}=\operatorname{det}\left(I+(\alpha-1) Z Z^{\prime}\left(I+\alpha P^{\prime} P\right)^{-1}\right) \\
& =\operatorname{det}\left(I+(\alpha-1) Z Z^{\prime}\left(I-\alpha P^{\prime} P+\left(\alpha P^{\prime} P\right)^{2}-\left(\alpha P^{\prime} P\right)^{3}+\ldots\right)\right) \\
& =\operatorname{det}\left(I+(\alpha-1) Z Z^{\prime}\right)
\end{aligned}
$$

and therefore

$$
\frac{\operatorname{det}(\alpha I+Q)}{\operatorname{det}\left(I+\alpha P^{\prime} P\right)}=\frac{\operatorname{det}\left(I+(\alpha-1) Z Z^{\prime}\right)}{\operatorname{det}\left(P P^{\prime}\right)}
$$

The determinant of $\left(I+(\alpha-1) Z Z^{\prime}\right)$ can be evaluated by means of its Eigenvalues. For any symmetric matrix $A \in \Re^{n}$, denote the vector of its Eigenvalues by $\Lambda(A)$. Its rank $r(A)$ gives the number of non-zero Eigenvalues. The vector of these non-zero Eigenvalues is denoted by $\Lambda^{+}(A) \in$ $\Re^{r(A)}$. The determinant of $A$ is equal to the product of its Eigenvalues.

We have

$$
\Lambda\left(I+(\alpha-1) Z Z^{\prime}\right)=\left(\begin{array}{c}
1 \\
1 \\
\cdot \\
1
\end{array}\right)+(\alpha-1) \Lambda\left(Z Z^{\prime}\right)
$$

Further, $r(Z)=2$ and $Z Z^{\prime}$ has rank 2 and two non-zero Eigenvalues of unity.

$$
\Lambda^{+}\left(Z Z^{\prime}\right)=\Lambda^{+}\left(Z^{\prime} Z\right)=\left(\begin{array}{l}
1 \\
1
\end{array}\right)
$$

In view of (80) we conclude that $\left(I+(\alpha-1) Z Z^{\prime}\right)$ has $T-2$ Eigenvalues of one and two Eigenvalues of $\alpha$. The determinant of $\left(I+(\alpha-1) Z Z^{\prime}\right)$ is the product of its Eigenvalues and we can write

$$
\operatorname{det}\left(I+(\alpha-1) Z Z^{\prime}\right)=\alpha^{2}
$$

Equation (79) reduces thus to

$$
\frac{\operatorname{det}(\alpha I+Q)}{\operatorname{det}\left(I+\alpha P^{\prime} P\right)}=\frac{\alpha^{2}}{\operatorname{det}\left(P P^{\prime}\right)}
$$

Taking logarithms and re-arranging terms yields

$$
\log \operatorname{det}(\alpha I+Q)=\log \operatorname{det}\left(I+\alpha P^{\prime} P\right)+2 \log \alpha-\log \operatorname{det}\left(P P^{\prime}\right)(84)
$$


Disreganding constants, the likelihood function (72) can be written as

$$
\mathcal{L}(x ; \alpha)=-\log \operatorname{det}\left(I+\alpha P^{\prime} P\right)-T \cdot \log R(\alpha)+(T+2) \log \alpha
$$

\section{A Moments Estimator For the Variances}

The likelihood estimation described in the preceding section lacks intuitive appeal, and its small-sample properties are difficult to ascertain. As an alternative, a moments estimator will be devised that is based on the idea that the calculated variances ought to be close to their expectations. (This type of estimator has originally been proposed by Schlicht (1989) in the context of state-space models.) idea has been used The estimator is derived by equating, at any sample size, the calculated variances with their expectations.

Assume a realization of a trend $y$ (that we can't observe) along with a realization of the time series $x$ (which is is taken as a realization of a random variable) for a given set of parameters $\beta, \sigma_{u}^{2}$, and $\sigma_{v}^{2}$. According to (44), this gives rise to the estimate $\hat{y}$ as a function of the variance ratio $\alpha=$ $\sigma_{u}^{2} / \sigma_{v}^{2}$ and of the time series $x$ which is the sum of trend $y$ and disturbance $u$ :

$$
\hat{y}=\left(I+\alpha P^{\prime} P\right)^{-1}(y+u)
$$

Since

$$
y=\left(I+\alpha P^{\prime} P\right) y-\alpha P^{\prime} P y
$$

and $v=P y$, equation (86) can be written as

$$
\hat{y}=y+\left(I+\alpha P^{\prime} P\right)^{-1}\left(u-\alpha P^{\prime} v\right)
$$

Pre-multiplication with $P$ gives

$$
\hat{v}=v+P\left(I+\alpha P^{\prime} P\right)^{-1}\left(u-\alpha P^{\prime} v\right)
$$

In a similar way, from $\hat{u}=x-\hat{y}$ we obtain

$$
\hat{u}=u-\left(I+\alpha P^{\prime} P\right)^{-1}\left(u-\alpha P^{\prime} v\right)
$$

Thus the estimated errors $\hat{u}$ and $\hat{v}$ are linear functions of the the normal random variables $u$ and $v$ :

$$
\left(\begin{array}{c}
\hat{u} \\
\hat{v}
\end{array}\right)=\left(\begin{array}{cc}
(I-M) & -\alpha M P^{\prime} \\
P M & I-\alpha P M P^{\prime}
\end{array}\right)\left(\begin{array}{l}
u \\
v
\end{array}\right)
$$




$$
\left(\begin{array}{l}
\hat{u} \\
\hat{v}
\end{array}\right)=\left(I-\left(\begin{array}{cc}
I & 0 \\
0 & P
\end{array}\right)\left(\begin{array}{cc}
M & M \\
M & M
\end{array}\right)\left(\begin{array}{cc}
I & 0 \\
0 & \alpha P^{\prime}
\end{array}\right)\right)\left(\begin{array}{l}
u \\
v
\end{array}\right) \quad(92)
$$

with

$$
M:=\left(I+\alpha P^{\prime} P\right)^{-1}
$$

and their joint distribution can be calculated:

$$
\left(\begin{array}{l}
\hat{u} \\
\hat{v}
\end{array}\right)=\mathcal{N}\left(0, \quad\left(\begin{array}{ll}
S_{11} & S_{12} \\
S_{21} & S_{22}
\end{array}\right)\right)
$$

with

$$
\begin{aligned}
S_{11} & :=\sigma_{u}^{2}(I-M)^{2}+\sigma_{v}^{2} \alpha^{2} M P^{\prime} P M \\
S_{12} & :=\sigma_{u}^{2}(I-M) M P^{\prime}-\sigma_{v}^{2} \alpha M P^{\prime}\left(I-\alpha P M P^{\prime}\right) \\
S_{21} & :=\sigma_{u}^{2} P M(I-M)-\sigma_{v}^{2} \alpha\left(I-\alpha P M P^{\prime}\right) P M \\
S_{22} & :=\sigma_{u}^{2} P M^{2} P^{\prime}+\sigma_{v}^{2}\left(I-\alpha P M P^{\prime}\right)^{2}
\end{aligned}
$$

From this, the expectation of the average squared errors can be calculated:

$$
\begin{aligned}
E\left\{\frac{1}{T} \hat{u}^{\prime} \hat{u}\right\}= & \sigma_{u}^{2} \cdot \frac{1}{T} \operatorname{tr}(I-M)^{2}+\sigma_{u}^{2} \alpha \frac{1}{T} \operatorname{tr}\left(M^{2} P^{\prime} P\right) \\
E\left\{\frac{1}{T-1} \hat{v}^{\prime} \hat{v}\right\}= & \sigma_{u}^{2} \cdot \frac{1}{T-1} \operatorname{tr}\left(M^{2} P^{\prime} P\right) \\
& +\sigma_{v}^{2} \cdot \frac{1}{T-1} \operatorname{tr}\left(I-\alpha P M P^{\prime}\right)^{2}
\end{aligned}
$$

Note that

$$
\begin{aligned}
\operatorname{tr}(I-M)^{2} & +\alpha \operatorname{tr}\left(M^{2} P^{\prime} P\right) \\
& =\operatorname{tr}(I-M)^{2}+\alpha \operatorname{tr}\left(M^{2} P^{\prime} P\right) \\
& =\operatorname{tr}\left(I-2 M+\left(I+\alpha P^{\prime} P\right) M^{2}\right) \\
& =\operatorname{tr}(I-M) \\
& =T-\operatorname{tr}(M)
\end{aligned}
$$

and 


$$
\begin{aligned}
\alpha \operatorname{tr}\left(M^{2} P^{\prime} P\right) & +\operatorname{tr}\left(I-\alpha P M P^{\prime}\right)^{2}= \\
& =\operatorname{tr}\left(\alpha M^{2} P^{\prime} P+I-2 \alpha M P^{\prime} P+\alpha^{2} M^{2}\left(P^{\prime} P\right)^{2}\right) \\
& =\operatorname{tr}\left(\alpha\left(I+\alpha P^{\prime} P\right) M^{2} P^{\prime} P+I-2 \alpha M P^{\prime} P\right) \\
& =\operatorname{tr}\left(I-\alpha M P^{\prime} P\right)
\end{aligned}
$$

Because

$$
M\left(I+\alpha P^{\prime} P\right)=I
$$

we have

$$
I-\alpha M P^{\prime} P=M
$$

Insering this into (102) gives

$$
\operatorname{tr}(I-M)^{2}+\alpha \operatorname{tr}\left(M^{2} P^{\prime} P\right)=\operatorname{tr}(M)
$$

and (99)-(100) reduce to

$$
\begin{aligned}
& E\left\{\hat{u}^{\prime} \hat{u}\right\}=\sigma_{u}^{2}(T-\operatorname{tr}(M)) \\
& E\left\{\hat{v}^{\prime} \hat{v}\right\}=\sigma_{v}^{2} \operatorname{tr}(M)
\end{aligned}
$$

The moments estimators for the variances, denoted by $\check{\sigma}_{u}^{2}$ and $\check{\sigma}_{v}^{2}$, are obtained by equalizing the estimated moments $\hat{u}^{\prime} \hat{u}$ and $\hat{v}^{\prime} \hat{v}$ with their expectations:

$$
\begin{aligned}
& \hat{u}^{\prime} \hat{u}=\check{\sigma}_{u}^{2}(T-\operatorname{tr}(M)) \\
& \hat{v}^{\prime} \hat{v}=\check{\sigma}_{v}^{2} \operatorname{tr}(M)
\end{aligned}
$$

Note that the estimated moments $\hat{u}^{\prime} \hat{u}$ and $\hat{v}^{\prime} \hat{v}$, as implied by (51)-(53) are functions of the observations $x$ and the variance ratio $\check{\alpha}=\check{\sigma}_{u}^{2} / \check{\sigma}_{v}^{2}$ and, thus, of the variances $\check{\sigma}_{u}^{2}$ and $\check{\sigma}_{v}^{2}$ and that the matrix $M$ depends on the variace ratio as well. Hence the solution to (108)-(109) amounts to finding a fixpoint.

The system can be written equivalently as 


$$
\begin{aligned}
\check{\sigma}_{u}^{2} & =\frac{1}{T}\left(\hat{u}^{\prime} \hat{u}+\alpha \hat{v}^{\prime} \hat{v}\right)=\frac{1}{T} R(\check{\alpha}) \\
\check{\sigma}_{v}^{2} & =\frac{\hat{v}^{\prime} \hat{v}}{\operatorname{tr}(M)}
\end{aligned}
$$

One way of estimating the variances is, thus, to find a fixpoint of (108)(109) or (110)-(111). Another way is the following.

Consider the function

$$
\mathcal{H}(x, \alpha)=-\log \operatorname{det}\left(I+\alpha P^{\prime} P\right)-T \cdot \log R(\alpha)+T \cdot \log \alpha
$$

The following Lemma states that the moments estimator can be derived by maximizing the function $\mathcal{H}(x, \alpha)$.

Lemma 1. The moments estimators, as defined by equations (110) and (111), can be obtained by minimizing the function $\mathcal{H}(x, \alpha)$ defined in (112) with respect to $\alpha$. The variances are computed from the minimizing value $\check{\alpha}$ as

$$
\begin{aligned}
\check{\sigma}_{u}^{2} & =\frac{1}{T} R(\check{\alpha}) \\
\check{\sigma}_{v}^{2} & =\frac{1}{T} \frac{R(\check{\alpha})}{\check{\alpha}}
\end{aligned}
$$

Proof. With $M=\left(I+\alpha P^{\prime} P\right)^{-1}$ we have

$$
\begin{aligned}
\frac{d \log \operatorname{det}\left(I+\alpha P^{\prime} P\right)}{d \alpha} & =\frac{d}{d \alpha}\left(T \log \alpha+\log \operatorname{det}\left(\frac{1}{\alpha} I+P^{\prime} P\right)\right) \\
& =\frac{1}{\alpha}(T-\operatorname{tr}(M))
\end{aligned}
$$

Note further that

$$
\begin{aligned}
R(\alpha) & =x^{\prime}(I-M)^{2} x+\alpha x^{\prime} M P^{\prime} P M x \\
& =x^{\prime}\left(I-2 M+M^{2}+\alpha M P^{\prime} P M\right) x \\
& =x^{\prime}\left(I-2 M+M\left(I+\alpha P^{\prime} P\right) M\right) \\
& =x^{\prime}(I-M) x \\
& =x^{\prime} x-x^{\prime} M x
\end{aligned}
$$


Consider

$$
\begin{aligned}
\frac{\partial M}{\partial \alpha} & =\frac{\partial\left(\alpha\left(\frac{1}{\alpha} I+P^{\prime} P\right)\right)^{-1}}{\partial \alpha} \\
& =-M P^{\prime} P M
\end{aligned}
$$

From (116) and (117) we obtain

$$
\begin{aligned}
R^{\prime}(\alpha) & =x^{\prime} M P^{\prime} P M x \\
& =\hat{v}^{\prime} \hat{v}
\end{aligned}
$$

Using these results, the derivative of $\mathcal{H}(x, \alpha)$ with respect to $\alpha$ is calculated as

$$
\frac{\partial \mathcal{H}}{\partial \alpha}=\frac{1}{\alpha} \operatorname{tr}(M)-T \frac{\hat{v}^{\prime} \hat{v}}{R}
$$

Substitutiong $\hat{v}^{\prime} \hat{v}$ by $\check{\sigma}_{v}^{2} \operatorname{tr}(M)$ and $\frac{R}{T}$ by $\check{\sigma}_{u}^{2}$ gives

$$
\frac{\partial \mathcal{H}}{\partial \alpha}=\frac{1}{\alpha} \operatorname{tr}(M)-\frac{\check{\sigma}_{v}^{2} \operatorname{tr}(M)}{\check{\sigma}_{u}^{2}}
$$

With $\alpha=\frac{\check{\sigma}_{u}^{2}}{\tilde{\sigma}_{v}^{2}}$, this reduces to zero. Hence any solution to (110), (111) satisfies the first-order condition for a maximum of $\mathcal{H}$. Conversely, (113) implies (110), and (111) can be derived by putting (119) to zero and substituting (114) into (119).

\section{The Relationship Between the MaXimum-Likelihood and the MOMENTS Estimator}

With the aid of the function $\mathcal{H}$, the asymptotic equivalence of the moments estimator and the maximum likelihood estimator can be established easily.

Theorem 3. The maximum likelihood estimator and the moments estimator are asymptotically equivalent.

Proof. We need to concentrate on the estimation of the smoothing parameter $\alpha$ because the formulae that link the variances to $\alpha$ are identical for the two estimators (See (74), (75) and (110), (111)).

Both the likelihood function $\mathcal{L}$ (eq. 85) and the criterion function $\mathcal{H}$ (eq. 112 ) tend to infinity with increasing $T^{2}$. In order to compare their asymptotic behavior, it is useful to divide both functions by $T$ and to compare the

${ }^{2}$ Note that log $\operatorname{det}\left(I+\alpha P^{\prime} P\right)$ is $O(T)$. 
moments criterion

$$
-\frac{1}{T} \log \operatorname{det}\left(I+\alpha P^{\prime} P\right)-\log R(\alpha)+\log \alpha
$$

with the likelihood criterion

$$
-\frac{1}{T} \log \operatorname{det}\left(I+\alpha P^{\prime} P\right)-\log R(\alpha)+\frac{T+2}{T} \log \alpha
$$

As $\frac{T+2}{T} \rightarrow 1$ for $T \rightarrow \infty$, both maximands are asymptotically identical and the theorem is proved.

Theorem 3 establishes that the moments estimator shares the attractive large-sample properties with the likelihood estimator. Conversely, it states that the intuitive interpretation of the moments estimator - that the calculated variances are equal to their expectations - carries over to the likelihood estimator in an approximate sense. This enhances the intuitive appeal of the maximum likelihood estimator.

\section{Notes on Numerical Performance}

A practical example is provided in Figure $1^{3}$. Reducing the variance ratio by one half or doubling it has no big effect on the qualitative behavior of the trend (Figure 2).

This does not tell much, however, about how well the method recovers the smoothing constant and the variances of the time series. Some simulations were conductued in order to obtain an impression about this aspect of performance. It is beyond the scope of the present paper to present a full-fledged Monte-Carlo study, however. The following remarks are intended to convey an overall impression.

The critical issue is, of course, how well (and how reliably) the smoothing constant is estimated by the moments estimator. Given the smoothing constant, the estimation is optimal anyway, and its theoretical distribution is known, see Theorem 1 and equation (48). It is, thus, sufficiuent to concentrate on the estimation of the smoothing constant $\alpha$, or rather, its logarithm $\log _{10}(\alpha)$, because this seems to be the more relevant quantity.

For the simulations done, the method works resonably well. The following describes just describe a few examples. Figure 3 depicts the frequency distribution for the estimates of the smoothing constant that are

\footnotetext{
${ }^{3}$ All computations are made using the Mathematica Package by Ludsteck (2004).
} 


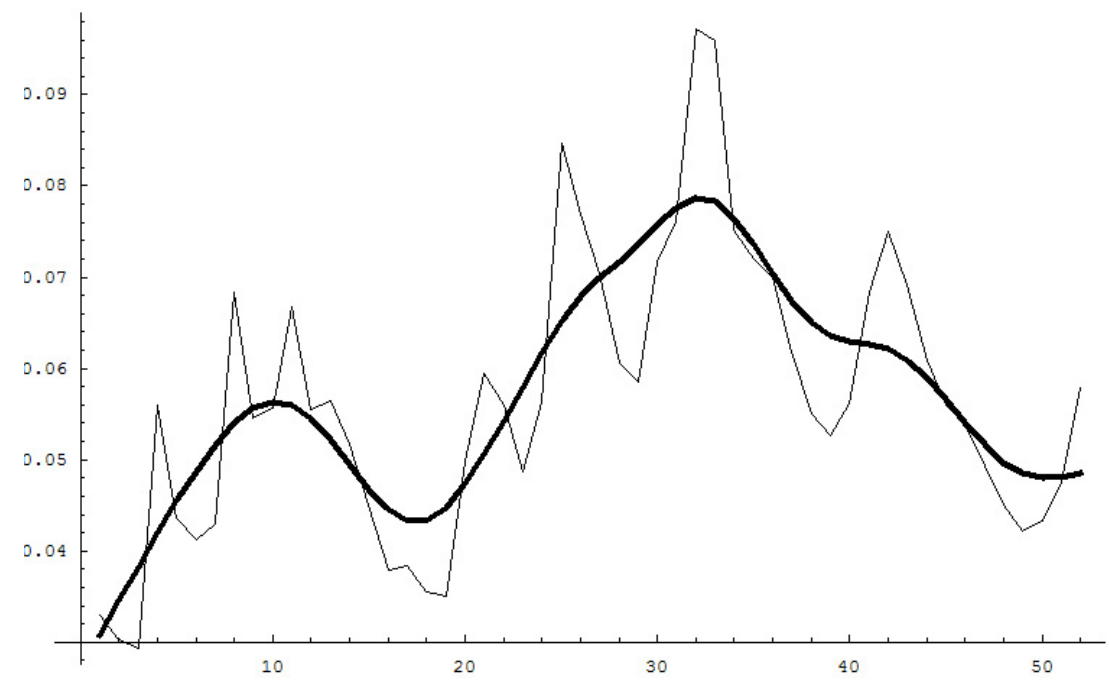

FIGURE 1. US unemployment 1951-2002, source: US Department of Commerce, Bureau of Labor Statistics. Estimated parameters: $\sigma_{u}^{2}=15, \sigma_{v}^{2}=.51$, and $\alpha=28.8$.

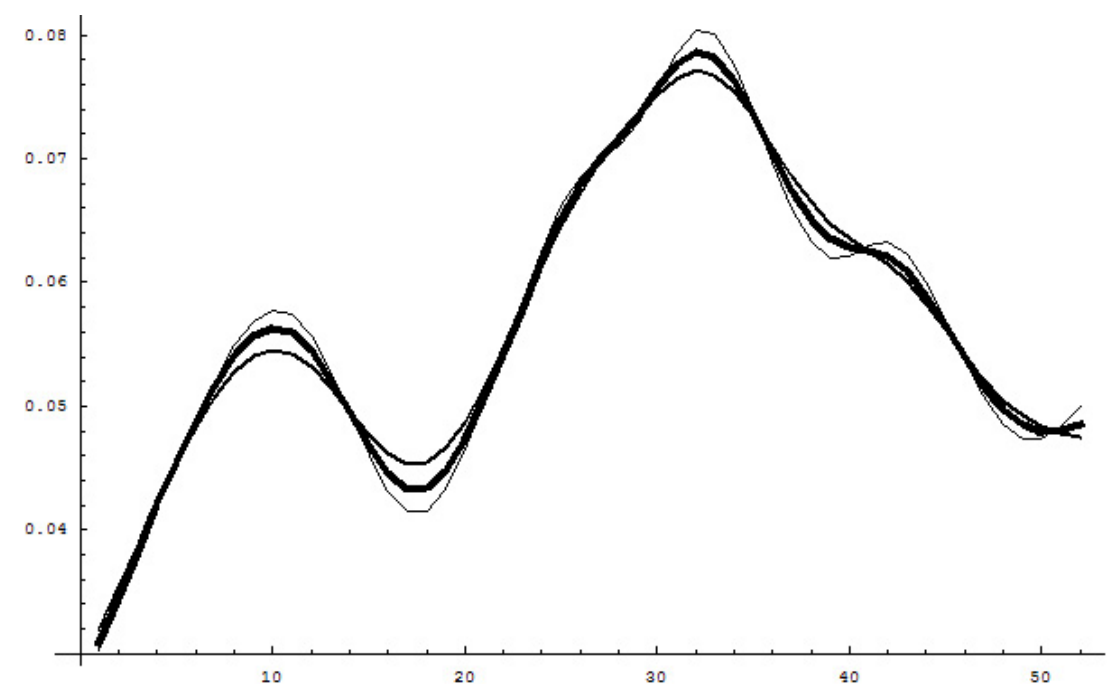

FIGURE 2. Effect of the smoothing constant: $\alpha=28.8$ (estimated value, heavily drawn curve), $\alpha=57.6$, and $\alpha=14.4$ (thin line). 

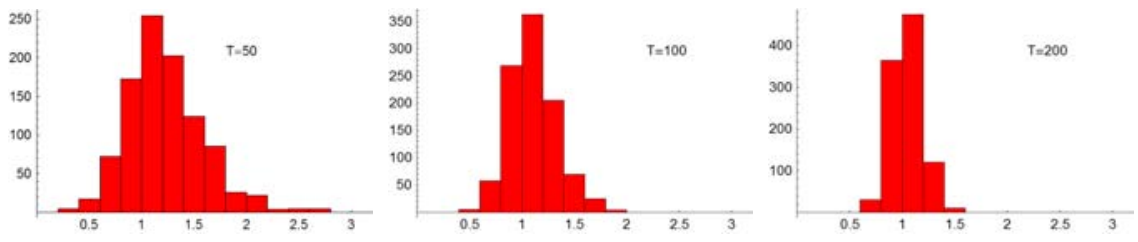

FIGURE 3. Distribution of estimates of $\log _{10}(\alpha)$ for time series of different length with $\sigma_{u}^{2}=10$ and $\sigma_{v}^{2}=1,1000$ trials each.

obtained by generating 1000 random series according to equations (1), (2), and (7) with variances $\sigma_{u}^{2}=10$ and $\sigma_{v}^{2}=1$ (corresponding to a smooting constant $\log _{10}(\alpha)=1$ ) for alternative lengths $T=50, T=100$, and $T=200$, respectively.

As expected, the estimates are less reliable for short series and more reliable for long series. ${ }^{4}$ It appears, further, that the median and the mean both tend to overrestimate the smoothing constant. With an increasing length of the series, the bias is reduced.

For long time series, the maximum-likelihood estimates and the moments estimates are nearly identical. For short time series, the difference is noticable. In these examples, the moments estimators work better in the sense of being less biased and pose less numerical difficulties. ${ }^{5}$

The decomposition depends on the smoothing constant, viz. the ratio of the variances, rather than the absolute magnitude of the variances (which are affected by scaling). This independence is reconfirmed in the simulations (Figure 5).

\footnotetext{
${ }^{4}$ The descriptive statistics of the simulations are given below, including the further case $T=$ 25 .

\begin{tabular}{c|ccccc}
$T$ & mean & median & min & max & standard deviation \\
\hline 25 & 1.36 & 1.33 & -0.89 & 2.71 & 0.50 \\
50 & 1.23 & 1.18 & 0.21 & 3.55 & 0.38 \\
100 & 1.11 & 1.08 & 0.42 & 1.91 & 0.22 \\
200 & 1.04 & 1.03 & 0.67 & 1.56 & 0.14
\end{tabular}

${ }^{5}$ For $T=20$, the problem is rather ill conditioned. Computations of the moments estimator failed to converge in $42 \%$ of the cases, as compared to $63 \%$ with the maximum-likelihood estimator. The correspondig failure rates for $T=50$ are $0.4 \%$ and $1.9 \%$, respectively.
} 

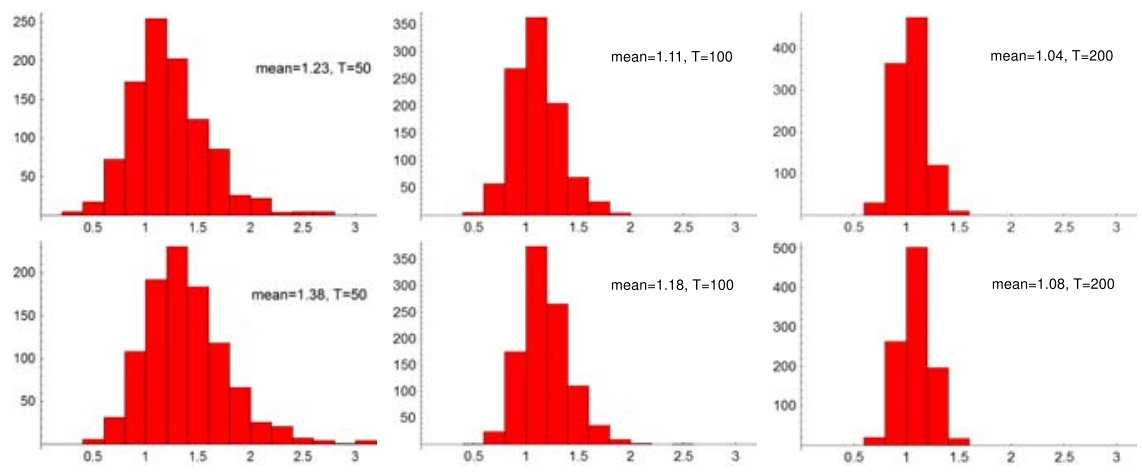

FIGURE 4. Comparison of moments estimators (first row) with maximum-likelihood estimators (second row), $T=$ 100, 1000 trials each.
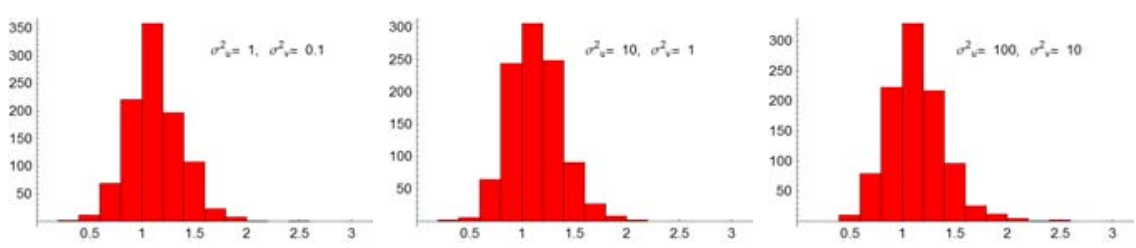

FIGURE 5. Simulations with identical variance ratios but different variances. The means are in the range $1.12-1.13$, and the standard deviations are in the range $0.24-0.27$ in all three cases. ( $T=80$ with 1000 trials each.)

Finally, Figure 6 gives the results when variance ratios are changed by a factor of 10. This shifts the distribution on the logarithmic scale to the left or to the right by one unit.

\section{NOTES ON MODELLING}

The trend filter discussed here has given rise to two strands of thought. One, originally proposed by Schlicht (1984) and also alluded to by Hodrick and Prescott (1997), relates to state-space modelling; the other, starting with King and Rebelo (1993), looks at performance in the frequency domain. The present paper falls into the first category.

The state-space literature tends to rely on Kalman filtering. As Kalman filters are one-sided filters, they are never efficient in the sense of using all 

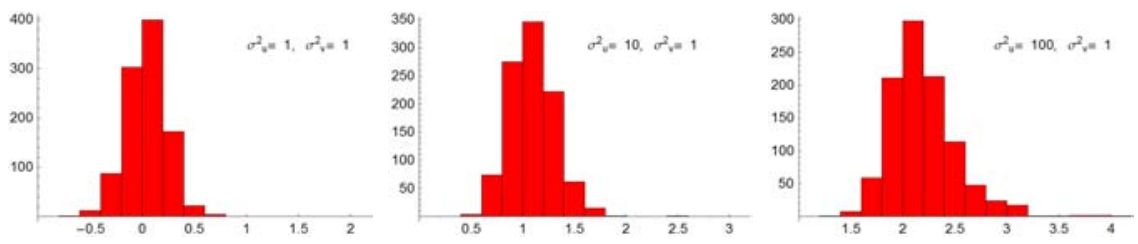

FIGURE 6 . Increasing the variance by some factor shifts the distribution of estimates to the right by the same factor. The means are $0.04,1.09$, and 2.19. The standard deviations are $0.19,0.22$, and 0.33 . ( $T=100,1000$ trials each, logarithmic scale.)

available information for estimating trend values at intermediate points in time. The filter proposed in Schlicht (1984) in the context of seasonal adjustment and detailed here for the case of a trend without seasonality is, in contrast, two-sided and uses all information available. This seems to be a preferable approach.

It seems misleading, however, to use the trend filter discussed here for quarterly or monthly data. This would require integrating the trend filter with a seasonal filter, as in Leser (1963) or Schlicht (1984) (preferably in the Schlicht/Pauly (1983) specification). Otherwise the seasonal pattern would misleadingly be interpreted as uncorrelated noise. For similar reasons, the filter is perhaps not adequate for identifying business cycles. The identification problem for business cycles is similar to the identification of seasonal patterns, but is considerably more involved, as business cycles vary in shape and periodicity.

The filter suggest some directions for future research. An improvement would be to develop a correction for the small-sample bias of the moments estimator. A further problem relates to the modelling and identification of business cycles.

\section{REFERENCES}

Henderson, R. 1924. A New Method of Graduation. Transactions of the Actuarial Society of America, 25, 29-40.

Hodrick, Robert J., and Prescott, Edward C. 1997. Postwar U.S. Business Cycles: An Empirical Investigation. Journal of Money, Credit, and Banking, 
29(1)(February), 1-16.

King, R. G., and Rebelo, S. T. 1993. Low Frequency Filtering and Real Business Cycles. Journal of Economic Dynamics and Control, 17, 207-31.

Leser, C. E. V. 1961. A Simple Method of Trend Construction. Journal of the Royal Statistical Society. Series B (Methodological), 23, 91-107.

Leser, C. E. V. 1963. Estimation of Quasi-Linear Trend and Seasonal Variation. Journal of the American Statistical Association, 58, 1033-43.

Ludsteck, Johannes. 2004. HPFilter. Mathematica Package. Wolfram Research, available at http://library.wolfram.com/infocenter/ MathSource/Mathematics/ ProbabilityStatistics/.

Schlicht, Ekkehart. 1981. A Seasonal Adjustment Principle and a Seasonal Adjustment Method Derived from This Principle. Journal of the American Statistical Association, 76(374)(June), 374-78. (Paper presented at the Econometric Society European Meeting Helsinki 1976, available at http://www.lrz.de/ ekkehart).

Schlicht, Ekkehart. 1984. Seasonal Adjustment in a Stochastic Model. Statistische Hefte, 25, 1-12. (available at http://www.lrz.de/ ekkehart).

Schlicht, Ekkehart. 1989. Variance Estimation in a Random Coefficients Model. Paper presented at the Econometric Society European Meeting Munich. (available at http://www. Irz.de/ ekkehart).

Schlicht, Ekkehart, and Pauly, Ralf. 1983. Descriptive Seasonal Adjustment by Minimizing Perturbations. Empirica. (available at http://www . lrz.de/ ekkehart).

Whittaker, E. T. 1923. On a New Method of Graduation. Proceedings of the Edinburgh Mathematical Society, 41, 63-75.

University of Munich, Department of Economics, Schackstr. 4, $80539 \mathrm{MU}$ NICH, GERMANY, SCHLICHT@LMU.DE 